\title{
Nosocomial pneumonia caused by water-born Legionella pneumophila in a pediatric hematopoietic stem cell transplantation recipient for thalassemia major
}

\author{
Tuğba Erat ${ }^{1 \oplus}$, Halil Özdemir $^{1 \oplus}$, Aysun Yahşi ${ }^{1 \oplus}$, Tuğçe Tural Kara ${ }^{1 \oplus}$, Elif Ünal İnce ${ }^{2 \oplus}$, \\ Kemal Osman Memikoğlu ${ }^{3 \oplus}$, Ergin Çiftçi ${ }^{1 \oplus}$, Erdal İnce ${ }^{1 \oplus}$ \\ Departments of ${ }^{1}$ Pediatric Infectious Diseases, ${ }^{2}$ Pediatric Hematology and Oncology and ${ }^{3}$ Infectious Diseases and Clinical Microbiology, \\ Ankara University Faculty of Medicine, Ankara, Turkey.
}

\begin{abstract}
Background. Nosocomial pneumonia caused by Legionella pneumophila serogroup 2-14 occurred in a 7-year-old patient following allogeneic hematopoietic stem cell transplantation for thalassemia major.

Case. The patient was diagnosed with nosocomial Legionella pneumophila by polymerase chain reaction (PCR) examination of the bronchoalveolar lavage and culturing Legionella pneumophila serogroup 2-14 from the patient's room faucet water. Legionella pneumophila was eradicated from our hospital's water distribution system by superheating and chemical eradication methods (hyper-chlorination and hydrogen peroxide). We did not detect any other case after this event.

Conclusion. Early recognition of contamination of the hospital water system with Legionella proves the importance of prevention in new cases.
\end{abstract}

Key words: Hematopoietic stem cell transplantation, Legionella pneumophila, pneumonia.

Legionella pneumophila is an intracellular gram-negative bacillus that requires special microbiological culture media. It seems that the risk of L. pneumophila pneumonia may be increasing in immunocompromised patients. ${ }^{1,2}$ Patients who receive bone marrow transplantation are sensitive to Legionella infection due to prolonged intervals of neutropenia and abnormalities in cell-mediated immunity. Therefore, Legionella infections in an immunocompromised patient can easily be severe and cause high mortality. ${ }^{3}$ Nosocomial Legionella infections are often transmitted via contaminated aerosol or aspiration of contaminated water. Relevant aerosol sources for hospital-acquired legionellosis include therapeutic devices and water distribution

Tuğba Erat

tugbacancan84@hotmail.com

Received 6th April 2017, revised 12th January 2020, 19th April 2020, accepted 26th April 2020. system outlets. ${ }^{4-6}$ Some practices for eradicating Legionella from the hospital water distribution system are superheating, hyper-chlorination, ultraviolet light, and the addition of copper and silver electrodes to the water., ${ }^{3,5}$ Herein, we report the management of an immunocompromised child with nosocomial Legionella pneumophila pneumonia acquired from the contaminated hospital water system.

\section{Case Report}

A 7-year-old girl followed-up with the diagnosis of thalassemia major (thalassemia mutation analysis IVS 1.110 G>A homozygote mutant) was hospitalized due to a second allogeneic hematopoietic stem cell transplantation (HSCT). The patient had undergone HSCT at the age of 4.5 years from her sibling with full compatible tissue type, but after three months, secondary graft rejection occurred, and recurrence of thalassemia major was considered. After three 
years, the patient underwent a $2^{\text {nd }} \mathrm{HSCT}$ again from her sibling with full compatible tissue type. Because of the patient's fever $\left(38.5 \mathrm{C}^{\circ}\right)$ during anti-thymocyte globulin (ATG) therapy administered in the period of HSCT preparation, intravenous (IV) cefoperazone-sulbactam therapy was initiated. Blood, catheter, and urine cultures collected from the patient. Fever's focus was negative, and her fever did not persist. Since expected engraftment was not achieved, the patient underwent bone marrow aspiration biopsy and chimerism examination. According to the results, the case was evaluated as primary rejection. Upon having a fever that exceeded $39 \mathrm{C}^{\circ}$ on the $26^{\text {th }}$ day after transplantation, blood, catheter, throat and urine cultures were collected, IV cefoperazone-sulbactam therapy was terminated and, IV meropenem and IV teicoplanin were initiated. Because the duration of neutropenia was prolonged, prophylactic fluconazole therapy was replaced with $1 \mathrm{mg} /$ $\mathrm{kg}$ IV liposomal amphotericin B therapy on alternate days. At follow-up, the patient had complaints of non-productive coughing, abdominal pain, and headache. On the 3rd day of fever, the respiratory sounds were decreased in the right middle and lower zones. A chest $\mathrm{X}$-ray examination was carried out, and pneumonic infiltration on the lower lobe of the

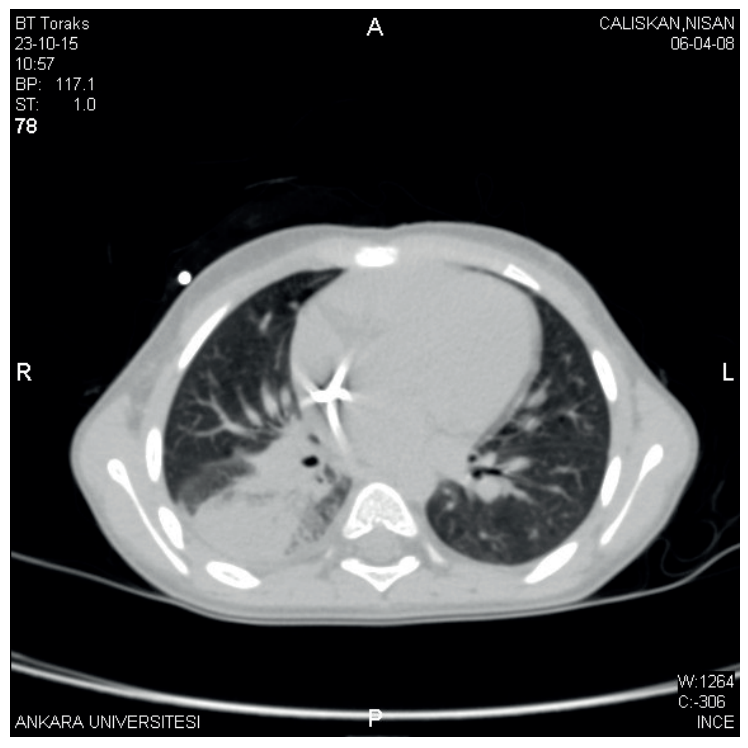

Fig. 1. Pneumonic infiltration of the lower lobe of right and left lung, chest X-ray 2. right lung was detected (Fig. 1). In this period, white blood cell (WBC) count, hemoglobin $(\mathrm{Hb})$ level, and platelets (Plt) count were found as $100 / \mathrm{mm}^{3}, 9.4 \mathrm{~g} / \mathrm{dL}$, and $17000 / \mathrm{mm}^{3}$, respectively and C-reactive protein (CRP) was $200 \mathrm{mg} / \mathrm{L}$. Serum galactomannan was found as $0.3 \mathrm{~s} / \mathrm{co}$ and cytomegalovirus (CMV) DNA was negative. Blood, catheter, urine, and throat cultures were also negative. Thoracic computerized tomography (CT) of the patient revealed a consolidated area in the right lung lower lobe superior segment and minimal pleural effusion (Fig. 2). Although the patient was under betalactam antibiotics treatment, fever persisted. Because of persistent fever, bronchoscopy was performed. Bronchoalveolar lavage (BAL) fluid was negative for bacterial and fungal cultures. BAL galactomannan level was found as 0.38 $\mathrm{s} / \mathrm{co}$, and rare leukocytes and lymphocytes were observed among alveolar macrophages. Infectious mononucleosis PCR, CMV PCR, Pneumocystis jirovecii PCR, Mycobacterium tuberculosis PCR, respiratory virus PCR panel were negative, while L. pneumophila PCR was positive. L. pneumophila antigen was negative in the urine. IV levofloxacin and PO azithromycin were added to her treatment for Legionella pneumonia. Autologous cells of the patient were infused because of long-term neutropenia and severe pneumonic infiltration. The patient's fever resolved on the $15^{\text {th }}$ day of fever, and clinical signs of pneumonia disappeared gradually. She received azithromycin for 14 days and levofloxacin for 21 days.

Upon L. pneumophila PCR being detected in the BAL fluid in this period, the case was reported to the local public health officials. Tap water of all floors, air conditioning systems, hot and cold water tank outlet temperatures, and chlorine levels were examined in our pediatric hospital. Cultures were collected for Legionella spp. Water temperature was between 39-68.4 $\mathrm{C}^{\circ}$, and the chlorine level varied between 0.26-0.47ppm. From the cultures collected, $12000 \mathrm{cfu} / \mathrm{L}$ colonies of L. pneumophila serogroup 2-14 was isolated from the culture of the tap water of the patient's room. Additionally, 550000cfu/L-9000000 cfu/L 


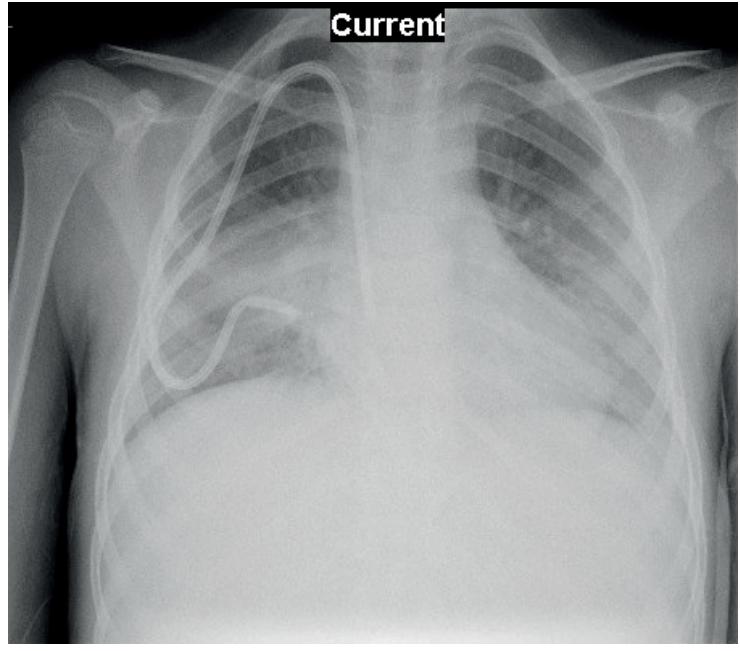

Fig. 2. Thoracic computerized tomography (CT) of the patient revealed consolidated area 3 and minimal pleural effusion in the right lung lower lobe superior segment.

colonies were isolated from the culture of the tap water of treatment room of neonatal intensive care unit (NICU) and the intermediate NICU.

Following the examination of L. pneumophila, superheating, hyper-chlorination, and hydrogen peroxide $\left(\mathrm{H}_{2} \mathrm{O}_{2}\right)$ were implemented for decontamination purposes. All faucets and showerhead filters were flushed with descaling agents. These processes were repeated two times with one-week intervals. Temperature, chlorine level, and culture samples were collected again from the same places after two weeks.

\section{Discussion}

We report nosocomial pneumonia of $L$. pneumophila in a 7-year-old female patient in the HSCT unit. Informed consent was obtained from the family. Because L. pneumophila was only detected with PCR in the patient's BAL fluid, we could not reveal its genotype. The increasing risk of L. pneumophila pneumonia in immunocompromised patients has previously been reported. ${ }^{1-4,8,9}$ In our patient, azithromycin, and levofloxacin were added to treatment without waiting for the results of water system culture samples since the patient was immunocompromised and did not give a clinical response to beta-lactam antibiotics. After detecting the PCR positivity, potential Legionella case was reported, and 22 samples were collected from the water systems. The water temperature of the tap water in the patient's room was found as $39 \mathrm{C}^{\circ}$ and chlorine level as $0.26 \mathrm{ppm} .12000 \mathrm{cfu} / \mathrm{L}$ colonies of L. pneumophila serogroup 2-14 was isolated from the culture of the tap water of the patient's room. In a study by Orsi et al. ${ }^{10}$ concerning Legionella control in the water system of antiquated hospital buildings, similar to our water characteristics, low level of residual free chlorine and water temperatures being between $20 \mathrm{C}^{\circ}$ and $45 \mathrm{C}^{\circ}$ were reported as risk factors. Nosocomial Legionella infections are often transmitted via contaminated aerosol or aspiration of contaminated water. Relevant aerosol sources for hospital-acquired legionellosis include humidifiers, nebulizer masks, showers, taps, cooling towers. ${ }^{4-6,11-13}$ In our case, we suggested that the infection was acquired by microaspiration of contaminated faucet water. L. pneumophila can create a biofilm layer, persisting for years in the water distribution system and requires biocides to be cleaned. ${ }^{12,14}$ Infection control for L. pneumophila is challenging and includes periodic inspections, cleaning, and maintenance of the water distribution systems, and decalcification of showers/taps. Routine microbiological surveillance and chemical monitoring of the water supply is necessary. ${ }^{10,12,15}$ For example, some practices for eradicating Legionella from the hospital water distribution system are superheating, hyper-chlorination, ultraviolet light, and the addition of copper and silver electrodes to the water. ${ }^{3,7}$ In our study, after the reproduction of $L$. pneumophila, the temperature of hot water was increased to 70 $\mathrm{C}^{\circ}$ for decontamination. Hyper-chlorination and $\mathrm{H}_{2} \mathrm{O}_{2}$ were applied in the water system and tanks. All faucets and showerhead filters were cleaned with decalcification. These processes were repeated two times with 1-week intervals. Temperature, chlorine level, and culture samples were collected again from the same places after two weeks and examined. Water temperature differed between 61.9-70 $\mathrm{C}^{\circ}$ and 
chlorine level between 0.36-0.67 ppm. Oren et al. ${ }^{3}$ also reported the control of the Legionella outbreak in their hospital, which was derived from the water system in the HSCT unit by superheating and hyper-chlorination. Likewise, the follow-up cultures collected in our hospital were negative, and the collection of water cultures was planned to take place once every two months for two years.

In brief, a nosocomial Legionella pneumonia outbreak originating from a water distribution system was prevented by early diagnosis, rapid treatment, and management of outbreak control with the collaboration of local public health officials. In order to prevent such outbreaks, inspections, cleaning, and maintenance of the water distribution systems, and decalcification of showers/taps should be performed periodically. There must be routine microbiological surveillance and chemical monitoring of the water supply in hospitals.

\section{REFERENCES}

1. Hayashi M, Kuraishi H, Masubuchi T, et al. A fatal case of relapsing pneumonia caused by Legionella pneumophila in a patient with rheumatoid arthritis after two injections of adalimumab. Clin Med Insights Case Rep 2013; 6: 101-106.

2. Heine S, Fuchs A, von Müller L, et al. Legionellosis must be kept in mind in case of pneumonia with lung abscesses in children receiving therapeutic steroids. Infection 2011; 39: 481-484.

3. Oren I, Zuckerman T, Avivi I, Finkelstein R, Yigla M, Rowe JM. Nosocomial outbreak of Legionella pneumophila serogroup 3 pneumonia in a new bone marrow transplant unit: evaluation, treatment and control. Bone Marrow Transplant 2002; 30: 175-179.

4. Osawa K, Shigemura K, Abe Y, et al. A case of nosocomial Legionella pneumonia associated with a contaminated hospital cooling tower. J Infect Chemother 2014; 20: 68-70.
5. Cordes LG, Wiesenthal AM, Gorman GW, et al. Isolation of Legionella pneumophila from hospital shower heads. Ann Intern Med 1981; 94:195-197.

6. Wadowsky RM, Yee RB, Mezmar L, Wing EJ, Dowling JN. Hot water systems as sources of Legionella pneumophila in hospital and nonhospital plumbing fixtures. Appl Environ Microbiol 1982; 43: 1104-1110.

7. Hoebe CJ, Kool JL. Control of legionella in drinkingwater systems. Lancet 2000; 355: 2093-2094.

8. Musallam N, Bamberger E, Srugo I, et al. Legionella pneumophila and Pneumocystis jirovecii coinfection in an infant treated with adrenocorticotropic hormone for infantile spasm: case report and literature review. J Child Neurol 2014; 29: 240-242.

9. Ampel NM, Wing EJ. Legionella infection in transplant patients. Semin Respir Infect 1990; 5: 3037.

10. Orsi GB, Vitali M, Marinelli L, et al. Legionella control in the water system of antiquated hospital buildings by shock and continuous hyperchlorination: 5 years experience. BMC Infect Dis 2014; 14: 394.

11. Franzin L, Scolfaro C, Cabodi D, Valera M, Tovo PA Legionella pneumophila pneumonia in a newborn after water birth: a new mode of transmission. Clin Infect Dis 2001; 33: e103-e104.

12. Bartley PB, Ben Zakour NL, Stanton-Cook M, et al. Hospital-wide eradication of a nosocomial Legionella pneumophila serogroup 1 outbreak. Clin Infect Dis 2016; 62: 273-279.

13. Yiallouros PK, Papadouri T, Karaoli C, et al. First outbreak of nosocomial Legionella infection in term neonates caused by a cold mist ultrasonic humidifier. Clin Infect Dis 2013; 57: 48-56.

14. Lin YE, Stout JE, Yu VL. Controlling Legionella in hospital drinking water: an evidence-based review of disinfection methods. Infect Control Hosp Epidemiol 2011; 32: 166-173.

15. Ditommaso S, Giacomuzzi M, Rivera SRA, Raso R, Ferrero P, Zotti CM. Virulence of Legionella pneumophila strains isolated from hospital water system and healthcare-associated Legionnaires disease in Northern Italy between 2004 and 2009. BMC Infect Dis 2014; 14: 483. 